\title{
Researches on the Duality Innovation Strategies for Scientific Small and Medium Enterprises
}

\author{
Lei Lei1 ${ }^{1,2,3,4}$, Wei Song1 \\ ${ }^{1}$ University of Science and Technology of China, Hefei, China \\ ${ }^{2}$ Southwest University of Science and Technology, Mianyang, China \\ ${ }^{3}$ Sichuan Civil-Military Integration Institute, Mianyang, China \\ ${ }^{4}$ International Science and Technology Strategy and Policy Research Center Sub-Center, Mianyang, China \\ Email: 380083844@qq.com
}

How to cite this paper: Lei, L. and Song, W. (2017) Researches on the Duality Innovation Strategies for Scientific Small and Medium Enterprises. Open Journal of Social Sciences, 5, 238-249.

https://doi.org/10.4236/jss.2017.59017

Received: September 4, 2017

Accepted: September 23, 2017

Published: September 26, 2017

Copyright (c) 2017 by authors and Scientific Research Publishing Inc. This work is licensed under the Creative Commons Attribution International License (CC BY 4.0).

http://creativecommons.org/licenses/by/4.0/

(c) (i) Open Access

\begin{abstract}
The realization path and mode selection issues for the duality innovation of the scientific small and medium enterprises (SMEs) haven't obtained a good settlement in China. With the questionnaire investigation and layered regression analysis for 292 scientific SMEs, this paper carried out researches on the realization path and mode selection for the duality innovation for scientific SMEs. The results show that relying purely on the interior path and correspondent paths to realize the duality innovation has no significant lifting functions for the enterprises' performance, relying purely on the exterior paths and correspondent modes to realize the duality innovation will pose harms to the enterprises' performance, and by taking the interactive paths between the interior path and exterior path to realize the duality innovation has significant lifting functions for the enterprises' performance. The duality innovation mode with the interior development innovation and the exterior exploration innovation under this path is lifting the enterprises' performance to the greatest extent.
\end{abstract}

\section{Keywords}

Duality Innovation, Path, Mode

\section{Introduction}

The scientific SMEs are one of the engines for the national innovation and the supporting strength for our country to establish the innovative country. But in the meantime, the dynamic and changeable market demands, the shortening 
technological service life and the fiercer competition home and abroad have got the balancing between the developing and explorative innovation to be the strategic topic influencing the survival and development of the scientific SMEs [1]. For this matter, the duality organization proposed the duality innovation that merges the development and exploration to solve this problem, which obtains the supports of large quantity of researches [2].

However, as the duality innovation involves the two innovative activities as development and exploration, which are totally different or forms a complete contrast, it needs special resources and norms to match with it. Therefore, it would lead to the resources competition, from which the organization tension will be caused. This competition and tension are more outstanding for the scientific SMEs [3]. Finding the realization path and mode for duality innovation suitable for scientific SMEs has become the urgent demand and focus for the current theories and practice researches [4].

While the existed researches' analysis and empirical verifications for the following core issues are still lacked: 1) What are the realization paths and modes for the duality innovation for scientific SMEs? 2) What influences do the different realization paths and modes for duality innovation have for the performance of the scientific SMEs? 3) Is there the best realization path and mode for the duality innovation for scientific SMEs?

In conclusion, based on the literature review, this paper summarized and formed the realization paths and correspondent modes for duality innovation. Then, it carried out analysis on the differential influences posed by the different paths and modes on the scientific SMEs, proposed the premium paths and modes hypothesis. At last, with the investigation data from 292 scientific SMEs, this paper carried out the empirical testing and concluded on the reflections from practices.

\section{Theories and Hypothesis}

\subsection{Theories Review}

The duality innovation is to carry out merging and balancing strategic arrangement for the developing and explorative innovations by inheriting the dialectical unity logic thinking [4].

The scholars in the early stage designed the realization path and modes for duality innovation from inside the enterprise, aiming to design the duality competition via reasonable organizational mechanism to allow development and explorations to be in the same enterprise [2]. For example, O'Reilly [5] et al. hold that the scientific SMEs should set the developing and explorative departments in different spaces at the same time to eliminate the conflicts between these two. Birkinshaw [6] et al. hold that cultivating the employees' duality thinking ability is the original path to realize the duality innovation for SMEs. Lubatkin [7] proposed that the integration of senior managers' team behaviors could help the enterprises to break the obstacles between the departments, realizing the mutual 
benefits and sharing between the departments, therefore breaking the resources and abilities bottleneck for the duality innovation of SMEs. Under this path, the duality innovation realization mode is presented as the attentions on the interior developing innovation and interior explorative innovation, as shown in Path 1 of Figure 1.

With the fluctuation of the macro environment and the increasing uncertainty of it, scholars' digging for the duality innovation's realization paths and modes turn to the external enterprises, trying to obtain and absorb the innovative factors from external organizations therefore obtaining the target of development or exploration. For example, Stadler [8] proposed that enterprises should be embedded into the social network, constructing the weak or strong relations with different network members to realize the equal emphasis on development and exploration. Lavie [9] pointed out consolidating the alliance that has been existing in the meantime of forging new alliances could break the contradiction of "development-exploration". Nagaoka [10] proposed that enterprises, by obtaining new technologies from outside in the meantime of transferring technologies to the outside through the means of technology franchising and cross licensing agreement, could realize the duality innovation. Under this path, the duality innovation realization mode is presented as the equal emphasis on the external developing innovation and external explorative innovation, as shown in Path 2 of Figure 1.

Currently, a few scholars have started to pay attentions to the duality innovation realization path with the interaction between inside and outside the enterprises. Stettner [11] et al. discovered that the mode of internal enterprises taking

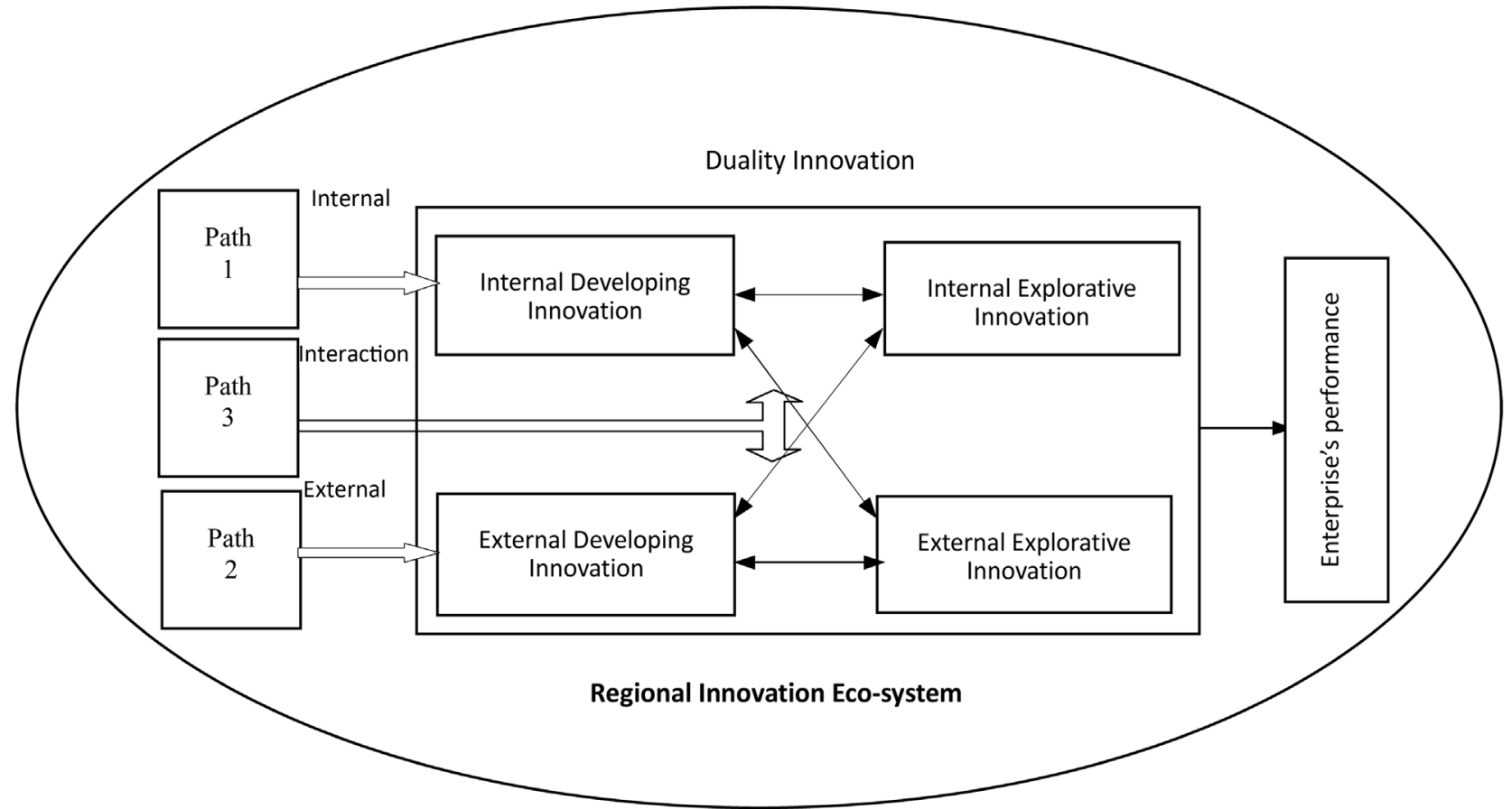

Figure 1. Research framework. 
charge of the developing innovation and the partner enterprises or the merged enterprises taking charge of the explorative innovation would improve the enterprises' performance to the maximum extent. Hoang [12] et al. pointed out the collaboration of the two experiences as the internal exploration and the external development would improve the performance of the project researched and developed. Lichtenthaler [13] proved that the technological fluctuation is the environmental reason why the enterprises employ the internal and external interactive way to realize the duality innovation. Under this path, the developing innovation and explorative innovation are decoupled on the single path and form the new coupling by crossing on the two paths as the internal path and external path of the enterprises. There are two types of realization modes for the duality innovation: one is the equal emphasis on the internal developing innovation and the external explorative innovation. The second one is the equal emphasis on the external developing innovation and the internal explorative innovation, as shown in Path 3 of Figure 1.

\subsection{Research Hypothesis}

\subsubsection{Enterprise's Internal Duality Innovation and Enterprise's Performance}

Developing and exploring the complementary coupling is the precondition for the duality innovation promotes the lifting of enterprise's performance [12]. While the scientific SMEs, as being restrained by the internal resources gift and the duality governing abilities, the two innovation activities are in the competition state or the loose coupling state [3]. In addition, the two types of innovations are with the self-enforcement nature, the duality innovation will build two comparatively closed systems that are hard for mutual supports therefore lead to the fact that the contrast between the development and exploration will exist forever, which will even enhance [11]. Even though this contrast could be eased through the internal system designing, the duality innovation inside the enterprise will cause the expansion of the organization, increase the difficulty for coordination and management and lift the costs for the multi-tasks conversion [13]. Besides the enterprises have to cut the rare resources to allocate to two types of innovations, which means that the scale effects generated by the concentrated resources couldn't be obtained. This is not only not beneficial for the core competitive edges building for scientific SMEs, but also it would lead it to the dilemma where it is not good at the exploration or development, that is, the so-called "double trap" of success and failure [11]. On this basis, this paper proposes the hypotheses:

H1: Relying purely on the internal enterprise to realize the duality innovation would pose harms to the performance of the scientific SMEs.

\subsubsection{The External Duality Innovation and Enterprise's Performance}

The Scientific SMEs obtain new innovative factors from the outside in the meantime transferring the current innovative results to the outside through the means 
of innovative network, alliance, merging, cross licensing and R\&D outsourcing, etc., which is the internal logic for the external duality innovation of enterprises [10]. However, as the scientific SMEs are generally in the non-leading position in the contractual and the non-contractual relations, its external developing innovation would need the exchange of a large quantity of autonomous knowledge assets and core technologies [11], and the quantity and quality of the innovative factors that the external explorative innovation could obtain are hard to reach the expected target. The unbalancing between the transfer and obtain of innovation would pose direct threats to its current core competition system. In the long run, it will lead the scientific SMEs to lose innovation motive and innovation abilities, lose the opportunities to dominate in the innovation, falling as the follower for innovation and allowing the large enterprises to occupy the major innovative benefits [14].

On this basis, this paper proposes the following hypothesis:

H2: Relying on the external enterprises to realize the duality innovation will harm the performance of the scientific SMEs.

\subsubsection{Enterprises' Internal and External Interactive Duality Innovation and the Performance of the Enterprises}

The internal and external interactive path in nature is that there is work division and collaboration of developing innovation and explorative innovation between enterprises. The work division of these two types of innovation gets the scientific SMEs to focus on specific innovation in the specific time duration, focus the utilization of rare resources, which will stimulate the scale economy, avoid the resources competition and organization tension, lower the conversion and coordination costs for conflicting tasks, form the uniform behavioral target, organization habits and the sequential learning environment [11]. While the collaboration of the two types of innovations guarantees the scientific SMEs to enjoy the complementary coupling benefits of development and exploration: the equal emphasis on the internal developing innovation and external explorative innovation would not only guarantee the sustainable and stable operation of the current business, but also avoid the danger of being locked in the certain state because of the lack of new knowledge. The equal emphasis on the external developing innovation and internal explorative innovation not only endows the enterprises wit high economic benefits (such as the transfer and licensing of innovation results) and intangible benefits (such as the enterprises' reputation and industrial status), but also guarantees the enterprises to maintain the flexibility to adapt to the environment changes in a timely manner.

To be more specific, how should these two innovations be distributed between the enterprises? This paper holds that the external organizations should undertake the explorative innovation while the scientific SMEs should engage in the developing innovation. And for the reasons: the explorative innovation requires the enterprises to surpass the priori knowledge and get rid of the path reliant. While the knowledge and knowledge embraced by the enterprises is with a high 
priori. In contrast, a variety and different resources could be obtained from outside the enterprises. With the push of the time, the enterprises could gradually change the original knowledge base, but the innovation opportunities pass very quickly, and the external enterprises is still the best means to obtain new knowledge and new technologies [4]. In the meantime, the explorative innovation requires the enterprises to shoulder high assets and more risks that would pose destructive reformation to the organization. These, undoubtedly, are gigantic challenges for the scientific SMEs [14]. At last, the external explorative innovation would generate "a variety of dilemmas" in the meantime of injecting rich foreign resources to the enterprises. Only by combining with the internal developing innovation will the enterprise draw the concept relation map between knowledge and meanings, therefore quickly realizing the internal and external connection and speeding up the commercial speed [4]. In conclusion, this paper proposed the following hypotheses:

H3: Compared with relying on purely on the internal and external enterprises, the internal and external interaction to realize the duality innovation would better promote the performance of the scientific SMEs.

H4: The equal emphasis on the internal developing innovation and the external explorative innovation would promote the performance of the scientific SMEs to the maximum extent.

\section{Researching Designs}

\subsection{Variable Measures}

The latent variables as the internal developing innovation (NK), internal explorative innovation (NT), the external developing innovation (WK) and external explorative innovation (WT) are measured by four items [13]: NK is measured by the digging, grasp, optimization and improvement by the current resources to the current technologies. NT is measured by the seeking, discovery, experimenting and breaking for the privately-owned resources for the new technologies. WK is measured by the frequency, depth, width and reliant for the external organization and current technologies. WT is measured by the frequency, depth, width and reliant for the enterprises to borrow from the external organization to seek, obtain and integrate new technologies. All the variables measurement takes the Likert-7 Measurement Method.

Given that the duality management mode regards the contradicted parties as the interdependent and inter-influential behaviors, this paper employs the multiplying item of NK, NT, WK and WT to measure the duality innovation mode [10].

The independent variable the enterprises' performance has designed with three items as the growth rate of sales income, pre-tax profits and market occupying rate.

The control variables include the enterprises' scale (GM), the region where is locates (SZD), and the type of ownership (SYQ). The GM measured with the 
employees number, and two virtual variables are introduced, that is, within 200 persons, between 201 and 500 and between 501 and 1000. As for the SZD, 1 represents the eastern area, and 0 represents the non-eastern area. For the SYQ, 1 is the code for domestically funded enterprises, and 0 is the code for non-domestically funded enterprises.

\subsection{Data Collection}

Paths for data collection: (1) 390 copies of questionnaires were granted to 39 enterprises in the national center cities as Chengdu city of Sichuan Province, Mianyang City, the scientific city of China and Deyang City, and 317 were recycled, with effective questionnaires of 295 copies. (2) 341 copies of questionnaires were issued to 3 partners (should be scientific SMEs) of the 39 enterprises above, 211 were recycled, with effective questionnaires of 193 copies. (3) With the EDP and EMBA resources of China University of Science and Technology and the MBA resources of Southwest University of Science and Technology, 224 questionnaires were issued, 221 copies were recycled, with the effective questionnaires of 219 copies, which were filled by the enterprises' senior managers or $\mathrm{R} \& \mathrm{D}$ project managers. The research grants questionnaires of 955 copies, among which 749 were recycled, and 707 were effective, with a recycle rate of $74 \%$. The questionnaire was covered from 2015 to 2016.

\subsection{Sample Characteristics}

The number of enterprises in the internet+, electronic information, new materials, new energy, light Electrical and mechanical integration, biology and medicine amounts to $33,69,27,25,49$ and 36 respectively, and 53 in other enterprises of the high tech domain. 41, 92 and 159 enterprises were in the eastern, middle and western areas respectively. 104, 117 and 71 enterprises were with a history of below 3 years, 3 to 5 years and above 5 years respectively. 238 and 54 enterprises were as the domestically funded ones and 54 as the non-domestically funded ones.

\section{Model Verifications}

\subsection{Credibility of the Scale and the Verifications of the Validity}

As shown in Table 1, all the Cronbach's Alpha coefficients of all the latent variables exceed the standard of 0.7 , showing that the scale maintains a high credibility.

The design of this scale is based on the mature scales abroad, for which the enterprises' senior managers and $\mathrm{R} \& \mathrm{D}$ project managers were invited to make the judgment and correction, to guarantee the validity of the scale's contents. As shown in Table 1: the combined credibility of all the latent variables surpasses 0.8 and AVEs are above 0.6, showing that the project measured maintains high convergence validity. As shown in Table 1 and Table 2, all the AVE mean square roots of the latent variable are above the relevant coefficient of other latent variables, showing that the latent variables maintains sound differentiating validity. 
Table 1 . Scale credibility and validity verification.

\begin{tabular}{cccc}
\hline Latent Variable & Cronbach' $\alpha$ & Combined Credibility & AVE \\
\hline NK & 0.916 & 0.917 & 0.734 \\
NT & 0.917 & 0.918 & 0.738 \\
WK & 0.908 & 0.901 & 0.693 \\
WT & 0.911 & 0.910 & 0.720 \\
JX & 0.912 & 0.898 & 0.747 \\
\hline
\end{tabular}

Table 2. The relevant coefficient of measured variables.

\begin{tabular}{cccccccccccc}
\hline Variable & Average & Standard & 1 & 2 & 3 & 4 & 5 & 6 & 7 & 8 & 9 \\
\hline $1 \mathrm{GM}$ & 0.25 & 0.43 & 1 & & & & & & & \\
$2 \mathrm{GM}$ & 0.37 & 0.47 & $-0.40^{* *}$ & 1 & & & & & & & \\
3 SZD & 0.40 & 0.49 & -0.03 & 0.04 & 1 & & & & & \\
$4 \mathrm{SYQ}$ & 0.71 & 0.45 & -0.07 & -0.04 & $-0.19^{* *}$ & 1 & & & & & \\
$5 \mathrm{NK}$ & 5.26 & 0.98 & 0.13 & -0.10 & 0.08 & -0.01 & 1 & & & & \\
$6 \mathrm{NT}$ & 5.62 & 0.93 & 0.07 & 0.00 & 0.02 & 0.04 & $0.70^{* *}$ & 1 & & & \\
$7 \mathrm{WK}$ & 5.31 & 1.04 & -0.06 & $0.19^{* *}$ & 0.07 & 0.04 & $0.20^{* *}$ & $0.19^{* *}$ & 1 & & \\
$8 \mathrm{WT}$ & 5.36 & 0.89 & 0.04 & $0.14^{*}$ & 0.00 & 0.03 & $0.33^{* *}$ & $0.37^{* *}$ & $0.55^{* *}$ & 1 & \\
$9 \mathrm{JX}$ & 3.88 & 0.68 & -0.09 & 0.08 & 0.06 & 0.10 & $0.52^{* *}$ & $0.53^{* *}$ & $0.20^{* *}$ & $0.31^{* *}$ & 1 \\
\hline
\end{tabular}

Note: ${ }^{*}$ shows that $\mathrm{p}<0.01 ;{ }^{*}$ shows that $\mathrm{p}<0.05$.

\subsection{Descritptive Statistics and Relevant Analysis}

It can be known from Table 2 that the relevant coefficients between the variables are below 0.7 , showing that the variables are rarely threatened by the multicollinearity. NK, NT, WK, WT maintain significant positive correlation with JX, providing the primary judgment basis for the further hypothesis and verifications.

\subsection{Regression Analysis}

Let's suppose that the verification employ the layered regression method: model 1 only introduced the control variables. Model 2 introduced four latent variables as NT, NK, WK and WT. Model 3 introduced the correspondent product term of four latent variables. To lower the impacts of multicollinearity, the average centralized processing is carried out to the data when the product term is involved.

As shown in Table 3, the VIF values for all the regression coefficients are lower than the critical value of 10 , and the regression results wouldn't be threatened by the multiple collinearity. Model 1 and Mode 2 provide basis for the verification for hypothesis. The F value of Model 3 is 3.102 and becomes significant under the situation where $\mathrm{p}<0.001$, showing that the Model 3's regression 
Table 3. Layered regression analysis.

\begin{tabular}{|c|c|c|c|}
\hline Variable & Model 1 & Model 2 & Model 3 \\
\hline \multirow{11}{*}{$1 \mathrm{GM}$} & & & -0.108 \\
\hline & & & 0.721 \\
\hline & & & 1.685 \\
\hline & & & 3.285 \\
\hline & & & 3.088 \\
\hline & -0.056 & -0.115 & 0.471 \\
\hline & & & 1.103 \\
\hline & & & 1.100 \\
\hline & & & 0.275 \\
\hline & & & -0.499 \\
\hline & & & 0.575 \\
\hline $2 \mathrm{GM}$ & 0.057 & 0.044 & 0.046 \\
\hline $3 \mathrm{SZD}$ & 0.075 & 0.040 & 0.042 \\
\hline $4 \mathrm{SYQ}$ & 0.115 & 0.092 & 0.098 \\
\hline $5 \mathrm{NK}$ & & $0.186^{* *}$ & $0.199^{* * *}$ \\
\hline $6 \mathrm{NT}$ & & $0.173^{* *}$ & $0.205^{\star *}$ \\
\hline $7 \mathrm{WK}$ & & 0.018 & 0.035 \\
\hline $8 \mathrm{WT}$ & & 0.101 & 0.086 \\
\hline $9 \mathrm{NK} \times \mathrm{NT}$ & & & 0.020 \\
\hline $10 \mathrm{WK} \times \mathrm{WT}$ & & & $-0.035^{\star \star}$ \\
\hline $11 \mathrm{NK} \times \mathrm{WT}$ & & & $0.248^{\star \star}$ \\
\hline $12 \mathrm{NT} \times \mathrm{WK}$ & & & $0.211^{*}$ \\
\hline $\mathrm{R} 2$ & 0.025 & 0.352 & 0.359 \\
\hline Adjusted R2 & 0.007 & 0.327 & 0.330 \\
\hline$\Delta \mathrm{R} 2$ & & $\begin{array}{c}0.302^{\star * *} \\
(\mathrm{p}=0.000)\end{array}$ & $0.007^{* * *}$ \\
\hline $\mathrm{F}$ & 1.355 & $13.923^{* * *}$ & $9.367^{* * *}$ \\
\hline VIF & $\leq 1.211$ & $\leq 2.513$ & $\leq 3.102$ \\
\hline
\end{tabular}

Note: ${ }^{* * *}$ shows that $\mathrm{p}<0.001,{ }^{* *}$ shows that $\mathrm{p}<0.01,{ }^{*}$ shows that $\mathrm{p}<0.05$.

equation has meanings. After adjustment, the $\mathrm{R}^{2}$ of model 3 is 0.359 . The changes of the dependent variable $35 / 9 \%$ can be explained by the independent variable of model 3, and the $\mathrm{R}^{2}$ of model 3 increases more significantly than Model 2, showing that independent variables have strong explanation effects for the enterprises' performance. As can be drawn from Model 3: (1) Relying purely on the internal path and the correspondent mode $(\mathrm{NK} \times \mathrm{NT})$ to realize the duality innovation has no significant influences on the performance of the scientific SMEs $(\beta=0.020, \mathrm{p}=0.783)$. The Hypothesis No.1 couldn't be supported. (2) Relying purely on the external path and the correspondent mode $(\mathrm{WK} \times \mathrm{WT})$ to realize the duality innovation has significant negative influences on the performance of the scientific SMEs $(\beta=-0.035, \mathrm{p}=0.018)$. The Hypothesis No. 2 is supported. (3) Relying on the two modes under the internal and external interactive path $(\mathrm{NK} \times \mathrm{WT} ; \mathrm{NT} \times \mathrm{WK})$ to realize the duality innovation has significant 
positive influences on the performance of the scientific SMEs $(\beta=0.248, \mathrm{p}=$ $0.007 ; \beta=0.211, \mathrm{p}<0.012$ ). The Hypothesis 3 is supported. (4) The relevance correlation between the $\mathrm{NK} \times \mathrm{WT}$ and the enterprises' performance is 0.248 , bigger than other relevant coefficients, showing that the duality innovation paying equal emphasis on internal developing innovation and the external explorative innovation would improve the performance of the scientific SMEs to the maximum extent. The Hypothesis 4 is supported.

\section{Conclusions and Reflections}

This paper carried out theoretical illustration and empirical verification surrounding through which means and modes the scientific SMEs to realize the duality innovation to improve the performance of the enterprises, and drew the following conclusions:

(1) Relying purely on the internal path to realize the duality innovation has no significant positive improvement functions for the scientific SMEs. Under the influence of the rare resources and self enforcement, the contradiction of development and exploration will exist in the scientific SMEs. Two sets of comparatively closed systems that are not compatible to each other would not only generate high innovation costs and risks, but also loses the scale economy effects of professional distribution.

(2) Relying purely on the external paths to realize the duality innovation has negative influences on the performance of the scientific SMEs. Given that the scientific SMESs' non-leading position in the contractual and non contractual relations and the limited abilities in innovation and learning. The result for borrowing the external enterprises to expand the duality innovation is to exchange for unequal innovative resources, opportunities and benefits in the meantime of transferring the autonomous intellectual property rights.

(3) Relying on internal and external interactive path to realize the duality innovation has significant improvement functions for the performance of scientific SMEs. Under the condition where there is the work-division and collaborative status between the developing innovation and explorative innovation, the scientific SMEs, on the one hand, focuses on the specific types innovation, therefore solving the contradiction between development and exploration from the root. On the other, it could enjoy the benefits from the complementary coupling of the two types of innovation, therefore laying the equal emphasis on stability and flexibility.

(4) The equal emphasis on developing innovation and external explorative innovation would improve the performance of the scientific SMEs to the maximum extent. This duality innovation mode meet with the knowledge base natures of the two types of innovations, guaranteeing that the scientific SMEs to lower the innovative risks while chasing the innovation benefits and promote the close coupling between the development and exploration, therefore speeding up the transformation and realization pace for innovation. 
The management reflections obtained in this paper are shown as follows: (1) The scientific SMEs couldn't pursue blindly for the duality innovation. The duality innovation, as the significant strategic arrangement mattering the existence and development of enterprises, must be based on the scientific and appropriate paths and modes. (2) Under the condition where the duality innovation path and mode couldn't effectively eliminate or ease the contradiction between development and exploration, the duality innovation, on the contrary, would pose harms to the performance of the enterprises. (3) The internal and external interactive duality innovation path would get the scientific SMEs "eased" in the bottleneck on resources gift, market status and learning abilities, etc., being the best path for the realization of its duality innovation. And the duality innovation mode laying the equal emphasis on internal developing innovation and the external explorative innovation could not only meet with the natures of the two types of innovations, but also get the two types of innovations to couple closely, therefore promoting the innovation process for the enterprises and lowering the risks and uncertainties for innovations. It is the optimum mode for the duality innovation of the scientific SMEs.

\section{Acknowledgements}

The work was supported by the soft science research project of Sichuan science and Technology Department (No. 2016ZR0119).

\section{References}

[1] Yu, F.F., Huang, Y.C. and Zhang, Y. (2013) Researches on the Balancing for "Exploration-Development" Innovation Path for Scientific SMEs. Scientific Progress and Countermeasures, 30, 79-83.

[2] Li, H., Chu, X.P. and Zheng, X. (2011) The Research Progress and Research Framework for Duality Innovation. Science Study and Science and Technology Management, 32, 58-66.

[3] Yang, X.R., et al. (2011) Would a Balanced Development of Developing Innovation and Explorative Innovation be Helpful for Improving the Performance of the Enterprises? Journal of Management Project, 25, 17-25.

[4] Voss, G.B. and Voss, Z.G. (2013) Strategic Ambidexterity in Small and Medium-Sized Enterprises: Implementing Exploration and Exploitation in Product and Market Domains. Organization Science, 24, 1459-1477. https://doi.org/10.1287/orsc. 1120.0790

[5] O’Reilly, C.A. and Tushman, M. (2008) Ambidexterity as a Dynamic Capability: Resolving the Innovator's Dilemma. Research in Organizational Behavior, 28, 185-206. https://doi.org/10.1016/j.riob.2008.06.002

[6] Gibson, C.B. and Birkinshaw, J. (2004) The Antecedents, Consequences, and Mediating Role of Organizational Ambidexterity. Academy of Management Journal, 47, 209-226. https://doi.org/10.2307/20159573

[7] Lubatkin, M.H., et al. (2006) Ambidexterity and Performance in Small Sized and Medium-Sized Firms: The Pivotal Role of Top Management Team Behavioral Integration. Journal of Management, 32, 646-672. 
https://doi.org/10.1177/0149206306290712

[8] Stadler, C., Rajwani, T. and Karaba, F. (2014) Solutions to the Exploration/Exploitation Dilemma: Networks as a New Level of Analysis. International Journal of Management Reviews, 16, 172-193. https://doi.org/10.1111/ijmr.12015

[9] Lavie, D., Kang, J. and Rosenkopf, L. (2011) Balance within and across Domains: The Performance Implications of Exploration and Exploitation in Alliances. Organization Science, 22, 1517-1538. https://doi.org/10.1287/orsc.1100.0596

[10] Nagaoka, S. and Kwon, H.U. (2006) The Incidence of Cross-Licensing: A Theory and New Evidence on the Firm and Contract Level Determinants. Research Policy, 35, 1347-1361. https://doi.org/10.1016/j.respol.2006.05.007

[11] Stettner, U. and Lavie, D. (2014) Ambidexterity under Scrutiny: Exploration and Exploitation via Internal Organization, Alliances, and Acquisitions. Strategic Management Journal, 35, 1903-1929. https://doi.org/10.1002/smj.2195

[12] Hoang, H.A. and Rothaermell, F.T. (2010) Leveraging Internal and External Experience: Exploration, Exploitation, and R\&D Project Performance. Strategic Management Journal, 31, 734-758.

[13] Lichtenthaler, U. (2012) Technological Turbulence and the Impact of Exploration and Exploitation Within and Across Organizations on Product Development Performance. Entrepreneurship Theory and Practice, 4, 979. https://doi.org/10.1111/j.1540-6520.2012.00520.x

[14] Wang, Y.D. and Li, J.H. (2012) The Construction of "Four Forces and Five Dimensions" for Duality Innovation Balancing System. Science Study and Science and Technology Management, 33, 173-180.

Submit or recommend next manuscript to SCIRP and we will provide best service for you:

Accepting pre-submission inquiries through Email, Facebook, LinkedIn, Twitter, etc. A wide selection of journals (inclusive of 9 subjects, more than 200 journals)

Providing 24-hour high-quality service

User-friendly online submission system

Fair and swift peer-review system

Efficient typesetting and proofreading procedure

Display of the result of downloads and visits, as well as the number of cited articles

Maximum dissemination of your research work

Submit your manuscript at: http://papersubmission.scirp.org/

Or contact jss@scirp.org 